\title{
Agreement in Reported Sexual Partnership Dates and Implications for Measuring Concurrency
}

\author{
DEVON D. BREWER, PHD, ${ }^{*}$ RICHARD B. ROTHENBERG, MD,† STEPHEN Q. MUTH, BA,‡ \\ JOHN M. ROBERTS, JR., PHD,§ AND JOHN J. POTTERAT, BA\|
}

\begin{abstract}
Objectives: To describe the reliability of reported dates of first and last sexual exposure, as elicited from sexually transmitted disease/ human immunodeficiency virus cases during routine contact investigation, and determine their adequacy for assessing concurrency.

Methods: We used contact tracing data from 5 studies in which both members of 774 dyads were interviewed and named each other as sex partners. We assessed partners' agreement on the dates of first and last exposure as related to precision (to the day, month, or year) of reported dates and demographic and behavioral characteristics of the dyad. We performed simulations that introduced reporting error, based on our observed data, to posited "true" temporal configurations of partnerships to assess the impact of unreliability in reporting on the
\end{abstract} measurement of concurrency.

Results: Thirty-two percent of dyads agreed on the exact date of first sexual exposure, and $36 \%$ did so for the date of last sexual exposure. Sixty-four percent agreed within 30 days on the date of first sexual exposure, and $81 \%$ did so for the date of last sexual exposure. The reliability of reported dates was positively related to the precision of the reports. Agreement on reported exposure dates was not meaningfully associated with any of the sociodemographic and behavioral variables available. Based on simulations, the positive predictive value of reported dates for estimating concurrency is approximately $\mathbf{8 0 \%}$ over a wide range of conditions.

Conclusions: These data suggest that the reliability of reported exposure dates is reasonably good but that estimating concurrency with reported dates is subject to some error. Data designed for the purpose and analyzed with adequate attention to the statistical and epidemiologic issues of assessing concurrency are needed.

IN A SEXUAL NETWORK, the timing of sexual partnerships constrains the potential flow of sexually transmitted pathogens. ${ }^{1,2}$ The concept of concurrency-cotemporal contact with more than 1 person-in sexually transmitted disease (STD)/human immunodeficiency virus (HIV) epidemiology has its underpinnings in a series of theoretical studies..$^{3-6}$ Simulations have suggested that a greater frequency of such partnerships in a population is associated with more rapid spread of disease. ${ }^{3,6}$ Such work has led to a more intensive consideration of the definition of concurrency, ${ }^{7,8}$ exploration of its ramifications, ${ }^{9-13}$ and mechanisms for its measurement. ${ }^{14}$

STD control programs have many decades' experience in eliciting the dates of first and last sexual exposure between persons interviewed and their contacts. ${ }^{15}$ Such reported dates are often

This work was supported, in part, by NIH/NIDA grant R01 DA12831 and NIH/NICHD grant R01 HD043678.

Correspondence: Devon D. Brewer, Interdisciplinary Scientific Research, P.O. Box 15110, Seattle, WA, 98115; E-mail: http://www.interscientific.net/ contact.html.
From *Interdisciplinary Scientific Research, Seattle, Washington; †Emory University School of Medicine, Atlanta, Georgia; $¥$ Quintusential Solutions, Colorado Springs, Colorado; §University of New Mexico, Albuquerque, New Mexico; and \|Colorado Springs,

Colorado

used to accomplish fundamental tasks in STD/HIV case investigation and partner notification (e.g., identifying partners at risk, prioritizing partners for intervention, classifying source and spread cases). Though not designed for the purpose, such data have also been used to assess concurrency by estimating the period of temporal overlap between partnerships. ${ }^{11,14}$ Several investigators have noted the dearth of research on the reliability and validity of reported partnership dates. ${ }^{14,16-18}$ In this assessment, we use data from 5 published studies based on contact tracing that permit evaluation of the agreement of sex partners on the dates of first and last sexual exposure.

\section{Methods and Materials}

The data for this assessment derive from a larger combined data set of network studies. ${ }^{19}$ We analyzed data from 5 contributing studies: (1) routine gonorrhea contact tracing in Colorado Springs in $1981^{20,21}$; (2) routine contact tracing for HIV in Colorado Springs between 1985 and 199922; (3) a study of chlamydia transmission and efficient interview (recall) periods in the context of routine contact tracing in Colorado Springs in 1996-199711,23; (4) a study of syphilis transmission involving contact tracing and social network investigation in Rockdale County, GA, in 1996199724; and (5) contact tracing focused on an outbreak of penicillinase-producing Neisseria gonorrhoeae (PPNG) in Colorado Springs between 1989 and 1991.25 For studies 1 and 5, interviewers elicited sexual partners for the 21 days before diagnosis plus the duration of symptoms for symptomatic cases and the 90 days before diagnosis for asymptomatic cases. For study 2 , interviewers elicited partners for the preceding 12 months, although sometimes the interview period extended farther back in time, depending on the circumstances of the case. The interview period for study 3 was 180 days before diagnosis. For study 4, the interview periods ranged from the 90 days before diagnosis (for primary syphilis cases) to the preceding 12 months or longer. Seventeen interviewers were involved in the 4 Colorado Springs studies (for the data we analyzed). Five interviewers participated in 1 study, 9 participated in 2 studies, and 3 participated in 3 or 4 studies; 7 interviewers accounted for $84 \%$ of the interviews included in analysis. 
The precise number of interviewers involved in study 4 (Rockdale County) is unknown but estimated to be approximately 10 to 15 .

In each data set, we identified dyads (partnerships) in which both persons had been interviewed as STD or HIV cases and had named each other as sexual contacts. Data were available on the dates of first and last exposure and frequency of sexual contact, as reported by each person in a dyad, as well as the age, sex, and race of each member of the pair and the dates of their interviews. The exact wording of questions for eliciting exposure dates was, in practice, not entirely standardized, although the meaning of the questions was constant across all interviews. Interviewers may also have varied in the precision of reported dates they sought and accepted.

We coded reported dates' level of precision according to conventions used by interviewers and data entry staff for recording imprecise dates in computerized databases. When a person reported only a year for an exposure date, it was recorded as July 1 or July 15 of that year. We treated all such dates as precise "to the year." When a person reported only a month and a year for an exposure date, it was recorded as the $15^{\text {th }}$ of that month. We treated all such dates as precise "to the month" and classified all other dates as precise "to the day," although some of these may actually have been reported only as precise to part of a month or week. For any level of precision, we treated the recorded exposure dates as literal dates in analysis (e.g., the absolute difference between June 15, 1990 [precise "to the month"] and July 1, 1990 [precise "to the year"] is 16 days). For most analyses, we classified dyads' level of precision by the less precise date reported by the 2 persons.

\section{Descriptive Statistics}

We determined the proportional distribution of precision for reported dates and the distributions of the differences between reported exposure dates within dyads by level of precision and characteristics of the dyad. We also computed $\gamma$ correlations between the levels of precision used by 2 persons in a dyad for a reported date.

Based on prior research on memory for autobiographical events, ${ }^{26}$ we examined whether respondents displayed heaping errors patterned by day of the week as indicated by the $\mathrm{H}$ statistic ${ }^{27}$ on multiples of 7 for absolute differences in reported exposure dates ( $\leq 30$ days). These analyses were restricted to dyads in which both persons reported a date precise "to the day." We indirectly investigated the possibility of forward telescoping (reporting temporally distant events as having occurred more recently than they actually did) of dates of first exposure by computing Pearson correlations between the signed difference in reported dates (first person interviewed - second person interviewed) and interval between the interview dates for persons in a dyad. If forward telescoping were present, negative correlations would be expected (with the second person interviewed in a dyad mentally shifting the date forward in time relative to the first person interviewed as a function of the interval between their interviews). Furthermore, we summarized the distribution of absolute differences in reported partnership duration within dyads and calculated the Pearson correlation between partners' reported durations within dyads.

\section{Multivariate Analysis}

To assess the independent association of dyad characteristics (heterosexual/homosexual, race concordance, age of each person, study/data source, frequency of sex, concordance in reporting sex frequency, whether the male partner was older [in heterosexual dyads], and interval between the pair's interviews) with differences in reported exposure dates, we used multiple regression models with "network autocorrelated" disturbances $28-30$ in which error terms are assumed to be correlated for partnerships that share a person.

\section{Simulating Concurrency}

We simulated the effect of unreliability in reported dates on measured concurrency using the actual distributions observed in this study. We used 2 different approaches-pair-based and distribution-based - to construct a pair of dyads, determine if they are concurrent (the "true condition"), alter the beginning and ending dates for both pairs according to reporting differences found in our empirical data, and reexamine whether they are still concurrent (the "observed" condition). We then calculated the sensitivity, specificity, positive predictive value (PPV) and concordance of the observed condition as a test of the true condition. We performed this simulation ( $\mathrm{N}=10,000$ trials) for all dyads in our data and subsets defined by precision of reporting and varied the "true" lag between partnerships (see Appendix for details).

\section{Results}

The 5 studies contributed 774 dyads that contained 1253 unique persons. Two hundred twelve persons were present in more than 1 dyad (range $=2-11$ ). In $74 \%$ of dyads, the pair was interviewed within 30 days of each other $($ mean $=57$ days, median $=10$ days, range $=0-5435$ ). We excluded dyads in which a person reported a date that occurred after his or her interview date (there were fully usable data on 723 dyads).

\section{Precision of Reporting}

In the 754 dyads with persons who both had valid reported dates of first exposure (1508 total reports), 840 reports (56\%) were precise to the day, 485 (32\%) were precise to the month, and 183 $(12 \%)$ were precise to the year. In contrast, in the 731 dyads with persons who both had valid reported dates of last exposure (1462 total reports), 1216 reports $(83 \%)$ were precise to the day, 215 reports $(15 \%)$ to the month, and $31(2 \%)$ to the year. The association between precision and recency of reported dates is also reflected in the correspondence within dyads in precision of reports for pairs who were interviewed within 7 days of each other (gammas $=0.77$ and 0.84 for dates of first and last exposure, respectively). Reports precise to a day were relatively uncommon for dates more than 365 days before the interview (18\% and 34\% of such reported dates of first and last exposure, respectively).

\section{Differences in Reported Dates of Exposure}

For dyads reporting to the day, the mean absolute difference in dates of first exposure was 21 days, and the median was 2 days (Table 1). The mean absolute difference in dates of last exposure for such dyads was 20 days, and the median was 2 days. For dyads reporting to the day, $84 \%$ agreed about the date of first exposure within 30 days ( $90 \%$ within 60 days), and $85 \%$ agreed about the date of last exposure within 30 days (92\% within 60 days). Agreement was substantially poorer for those dyads with lower precision. Using all levels of precision, 64\% agreed about their first exposure within 30 days (72\% within 60 days), and $81 \%$ agreed about their last exposure within 30 days (88\% within 60 days). Figures 1 and 2 show histograms of the full distributions for differences in reported first and last exposure dates. Agreement on reported dates of last exposure increased somewhat $(n=603,88 \%$ agreed within 30 days) after omitting dyads in which one person reported a date of last exposure falling after the other's interview date. 
TABLE 1. Agreement Between Persons in a Dyad on Dates of First and Last Exposure*

\begin{tabular}{lcc}
\hline $\begin{array}{l}\text { Difference } \\
\text { Between Dates }\end{array}$ & $\begin{array}{c}\text { First Exposure, } \\
\text { N (\%) }\end{array}$ & $\begin{array}{c}\text { Last Exposure, } \\
\text { N (\%) }\end{array}$ \\
\hline All dyads & & \\
Exact agreement & $242(32)$ & $264(36)$ \\
Within $30 \mathrm{~d}$ & $482(64)$ & $590(81)$ \\
Within $60 \mathrm{~d}$ & $542(72)$ & $644(88)$ \\
Level of precision & Days & Days \\
Mean difference & & \\
To the day & 21 & 20 \\
To the month & 113 & 46 \\
To the year & 400 & 207 \\
All & 122 & 32 \\
Median & & \\
$\quad$ difference & 2 & 2 \\
To the day & 26 & 91 \\
To the month & 137 & 3 \\
To the year & 13 & \\
All & & \\
\hline
\end{tabular}

*For date of first exposure, 323 dyads were precise to the day, 303 were precise to the month, and 128 were precise to the year. For date of last exposure, 549 dyads were precise to the day, 156 were precise to the month, and 26 were precise to the year. The precision of a dyad was determined by the less precise exposure date reported by the 2 persons.

\section{Heaping and Telescoping}

For dyads in which both persons reported to the day and differences in reported dates were $\geq 30$ days, there was modest, nonsignificant heaping on multiples of 7 for absolute differences in dates of first exposure $\left(\mathrm{H}_{1}=0.31, P=0.20\right)$ but none for differences in dates of last exposure $\left(\mathrm{H}_{1}=-0.03, P=0.46\right)$. We did not detect any forward telescoping in reported dates of first exposure. The correlation between the signed difference in reported dates and interval between interview dates in a dyad was 0.00 for all levels of reporting precision ( $\mathrm{n}=754$ dyads) and -0.02 for dyads reporting to the day or month $(n=626)$.

\section{Duration of Partnerships}

Fifty-seven percent of reported partnerships had a duration of $\leq 90$ days, and the distribution was strongly skewed to the right

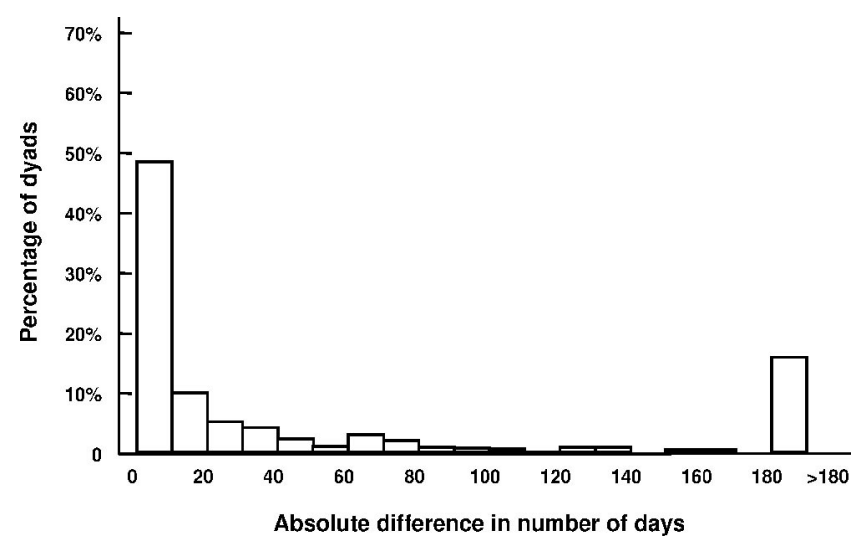

Fig. 1. Histogram of absolute differences in reported dates of first exposure, all levels of precision.

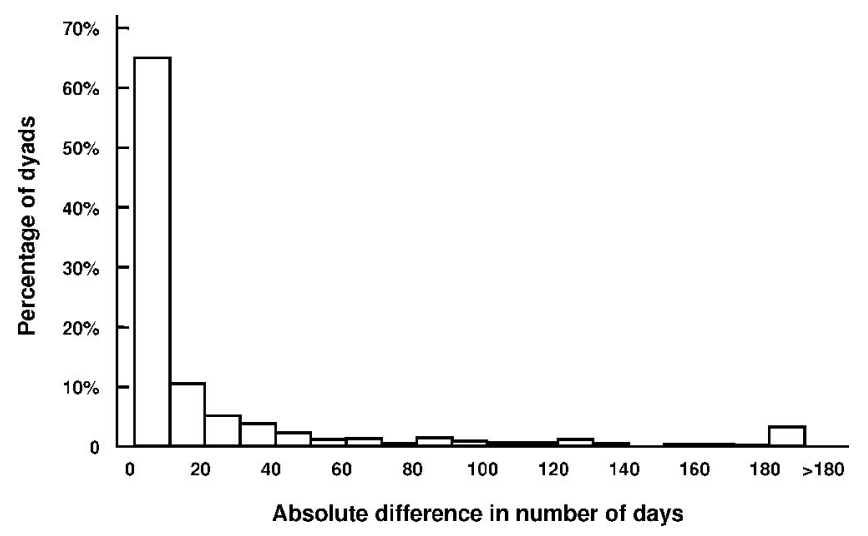

Fig. 2. Histogram of absolute differences in reported dates of last exposure, all levels of precision.

$(\mathrm{n}=1446$ reports, mean $=323$ days, median $=60$ days, range $=$ $1-6593$ days, and skewness $=3.8$ ). The reported durations tended to be similar within dyads $(r=0.87$ for all dyads, $r=0.73$ for those in which both reported durations $<366$ days). The distribution of absolute differences in reported duration within dyads was also strongly skewed to the right, with a mode of 0 days, a median of 13 days, a mean of 120 days, a standard deviation of 317 days, and a skewness of 5.8.

\section{Dyad Characteristics Associated With Reliability of Reporting}

In bivariate comparisons (Table 2) and multiple regression models, the only dyad characteristics that were consistently, albeit modestly, related to reliability of reported dates were those that effectively represented measurement factors. The interval between interviews in a dyad (for those with $<200$-day intervals) was slightly positively correlated with differences in reported dates of first exposure $(\mathrm{r}=0.10, P<0.01)$ and last exposure $(\mathrm{r}=0.07$, $P=0.08)$. When the full range of interview intervals was included, these correlations increased, due to the influence of outliers (data not shown). Interview interval had a significantly positive association with differences in reported dates in most multivariate models, but results were sensitive to model specification and removal of HIV contact tracing dyads (data not shown).

Dyads identified from HIV contact tracing had lower reliability than other dyads. Persons in HIV dyads were more likely to report exposures in the distant past than persons in other dyads. The HIV cases had longer interview periods (34\% of reported dates of last exposure in HIV dyads were $>180$ days before the interview versus $3 \%$ in the other dyads) and longer intervals between interviews for dyad pairs (HIV dyads: mean $=338$ days, median $=49$ days; other dyads: mean $=24$ days, median $=9$ days . Consequently, persons in the HIV dyads tended to report dates precise to the month or year for first and last exposures $(91 \%$ and $76 \%$, respectively), more so than persons in the other studies $(53 \%$ and $21 \%$, respectively).

Dyads that agreed about the frequency of sex (single versus multiple occasions) reported exposure dates more reliably than dyads that disagreed, though differences for the latter were small. These limited differences in reported dates for dyads discordant on reported frequency were a consequence of the relatively short duration of these partnerships (as defined by the person reporting multiple exposures; median $=29$ days versus median $=112$ days for other dyads). That is, in such dyads, the person reporting multiple exposures tended to report first and last exposure dates that were close together in time, which restricted the degree of 
TABLE 2. Percentage Agreement Between Partners on Date of First and Last Exposure, by Dyad Characteristics (All Levels of Precision)

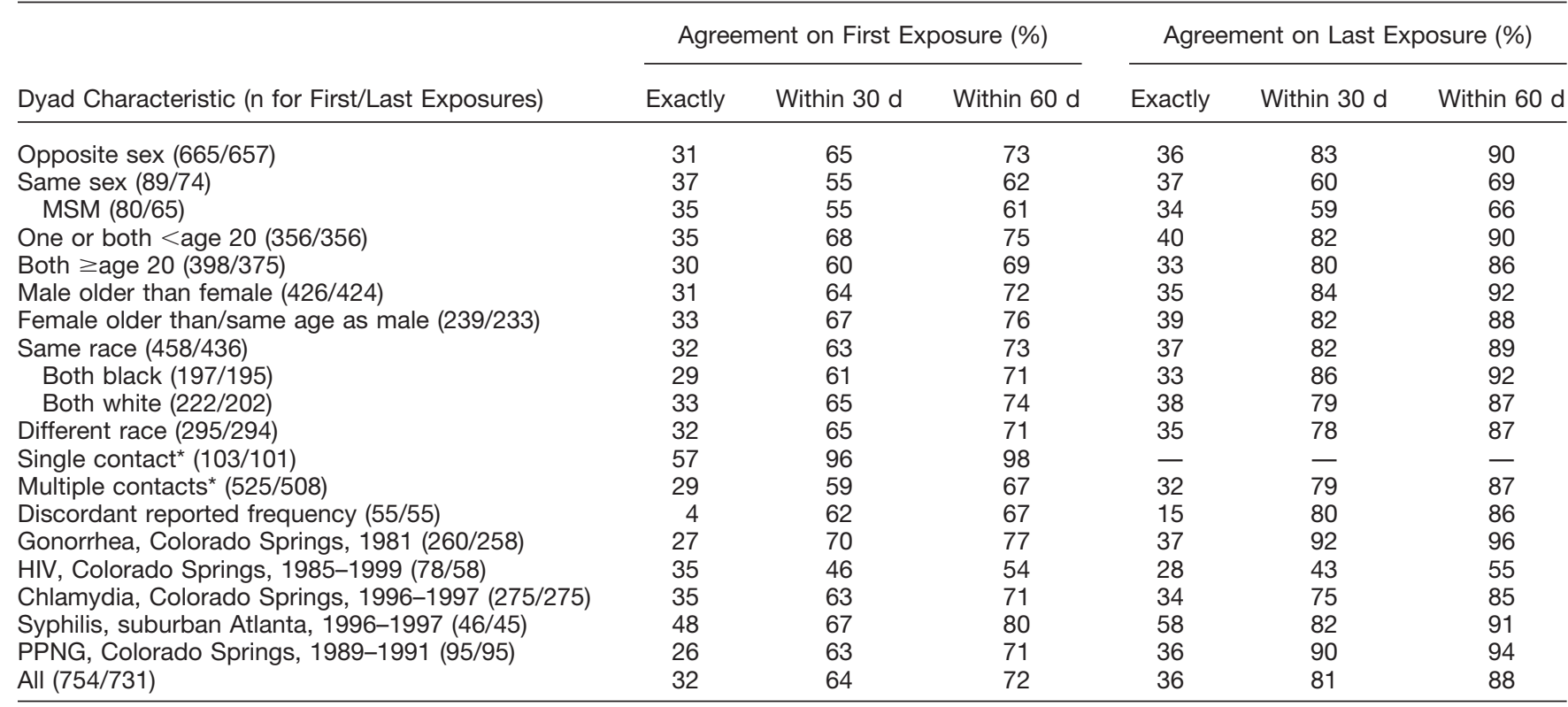

*Dyads in which both persons agreed on frequency (single versus multiple occasions).

difference between partners in reported exposure dates, even though agreement was rarely exact.

Demographic characteristics of dyads (age, sex of older partner, age difference, racial similarity, and behavioral sexual orientation) were not meaningfully and consistently related to differences in reported dates of exposure (Table 2 and data not shown). The apparent bivariate association between same sex dyads and greater unreliability disappeared in multivariate models and was accounted for by study source (HIV contact tracing) (data not shown). Sixty-one percent (57/94) of all homosexual dyads were from the HIV study. In all multiple regression models, the term for network autocorrelation was negligible and nonsignificant (data not shown). That is, the regression disturbance terms were not more similar in dyads that shared an individual than in dyads that did not. This may suggest that unmeasured individual (rather than dyadic) factors were not related to interpartner reliability of reported dates. The low $r^{2}$ values $(0.04-0.14)$, though, suggest that important predictors of reliability of reported dates may have been absent from these data.

\section{Simulating Concurrency}

The 2 simulation approaches we used were in substantial agreement. In the pair-based approach, sensitivity varied from $71 \%$ to $80 \%$, specificity from $65 \%$ to $93 \%$, and PPV and concordance from $70 \%$ to $90 \%$ (Table 3). For the distribution-based approach, the sensitivity varied from $83 \%$ to $92 \%$, the specificity from $73 \%$ to $93 \%$, and the PPV from $77 \%$ to $90 \%$ (data not shown). Both approaches also showed that, at any level of precision, as the lag between partnerships increases, prevalence of concurrency declines; sensitivity, specificity, and concordance increase; and PPV decreases because of the influence of prevalence.

\section{Discussion}

STD control staff have queried patients about dates of first and last exposure with their sexual contacts for about 5 decades. The standard interview form (page PS-3715) on which such information is recorded has changed little over the years. Despite the wealth of anecdotal experience (the corroboration of dates provided by patients and their contacts), the quality of such data has not been formally evaluated. The recognition of concurrency's importance in transmission of STD/HIV reinforces the need for such evaluation.

The reliability of reported partnership dates has been the subject of only limited study, however. Married, predominantly monogamous couples in Senegal and Thailand displayed high interpartner reliability for reported dates of last sexual intercourse (Senegal: $72 \%$ concordant within 1 day; Thailand: $64 \%$ concordant within 3 days). ${ }^{31,32}$ Fourteen percent of Dutch STD cases and partners they referred for examination agreed exactly on the date of first sex, $41 \%$ agreed within the same month, and $82 \%$ agreed within the same year. ${ }^{33}$ In addition, dyads of drug injectors in New York City displayed strong interpartner concordance $(r=0.89)$ in the reported duration of their sexual partnerships (measured in months). ${ }^{34}$

In our study, we used data from standard STD control program contact tracing and found that concordance between partners in reporting dates of first and last exposures and partnership durations was reasonably good but clearly varied by the precision with which partners reported dates. More recent exposures tended to be reported more precisely and reliably than less recent exposures, as suggested by the associations between reliability and the length of recall period and the interval between interviews for persons in a dyad. This result is consistent with research on the accuracy of reported dates of autobiographical events. ${ }^{26,35-39}$ Apart from these measurement factors, we did not find evidence that individual or dyadic characteristics were associated with reliability of reported dates, although our design makes our assessment of individuallevel factors indirect.

The absence of telescoping and consistent heaping suggests reported dates of exposure are largely free of these 2 common types of response error. The lack of telescoping may be due to lack of a temporal boundary in the elicitation of most exposure dates, since such boundaries tend to induce telescoping. ${ }^{26}$ The weak and 
TABLE 3. Results From Simulations Using Pair-Based Approach (See Appendix): Sensitivity, Specificity, Positive Predictive Value (PPV), and Concordance of Observed Concurrency Compared to True Concurrency*

\begin{tabular}{lrccccc}
\hline Precision & Lag & Prevalence & Sensitivity & Specificity & PPV & Concordance $^{\dagger}$ \\
\hline Day & $\pm 10 \mathrm{~d}$ & 0.61 & 0.71 & 0.76 & 0.82 & 0.73 \\
Day & $\pm 30 \mathrm{~d}$ & 0.46 & 0.74 & 0.82 & 0.78 & 0.78 \\
Day & $\pm 60 \mathrm{~d}$ & 0.36 & 0.78 & 0.87 & 0.77 & 0.84 \\
Day & $\pm 90 \mathrm{~d}$ & 0.31 & 0.78 & 0.90 & 0.77 & 0.86 \\
Day & $\pm 180 \mathrm{~d}$ & 0.22 & 0.80 & 0.93 & 0.77 & 0.90 \\
Day/Mo & $\pm 10 \mathrm{~d}$ & 0.75 & 0.71 & 0.68 & 0.87 & 0.70 \\
Day/Mo & $\pm 30 \mathrm{~d}$ & 0.65 & 0.71 & 0.74 & 0.83 & 0.72 \\
Day/Mo & $\pm 60 \mathrm{~d}$ & 0.58 & 0.74 & 0.80 & 0.84 & 0.77 \\
Day/Mo & $\pm 90 \mathrm{~d}$ & 0.53 & 0.75 & 0.82 & 0.82 & 0.78 \\
Day/Mo & $\pm 180 \mathrm{~d}$ & 0.42 & 0.76 & 0.87 & 0.80 & 0.82 \\
All & $\pm 10 \mathrm{~d}$ & 0.78 & 0.71 & 0.65 & 0.88 & 0.70 \\
All & $\pm 30 \mathrm{~d}$ & 0.70 & 0.72 & 0.70 & 0.85 & 0.72 \\
All & $\pm 60 \mathrm{~d}$ & 0.63 & 0.73 & 0.76 & 0.84 & 0.74 \\
All & $\pm 90 \mathrm{~d}$ & 0.58 & 0.74 & 0.79 & 0.83 & 0.76 \\
All & $\pm 180 \mathrm{~d}$ & 0.49 & 0.75 & 0.84 & 0.82 & 0.80 \\
\hline
\end{tabular}

*Each row reports a simulation with $\mathrm{N}=10,000$ trials. The subgroups that provided the distributions are defined by 2 variables: (1) precision, the precision with which the dates of first exposure were reported (see text); (2) lag, the maximum extent by which the lengths of the dyadic partnerships are offset (XQ in the Appendix). For example, the simulation reported in the first row used dyads wherein both partners reported precision to the day, and a lag of up to $10 \mathrm{~d}$ was assigned. The simulation in the last row was drawn from all 723 observed dyads with valid dates of first and last exposures and used a lag of up to $180 \mathrm{~d}$.

${ }^{\dagger}$ Defined as the proportion of trials that resulted in true positives or true negatives.

inconsistent observed heaping indicates that cases tended not to remember the day of week for an exposure if they reported the date erroneously (or at least unreliably).

Cases and partnerships in our data may not be representative of the spectrum involved in the transmission of STD/HIV, which may limit the generalizability of our findings. Our research design also prevents conclusions on the validity of reported exposure dates. Nonetheless, the relatively high reliability of reported exposure dates, the apparent absence of telescoping, and others' observations of greater accuracy in dating personal events involving boyfriends/girlfriends or sex (relative to other types of personal events $)^{26}$ suggest that reported dates of sexual exposures may be fairly accurate.

Although the observed level of reliability in reported dates of first and last sexual exposures is moderately good, our simulation indicates that it is not sufficient to avoid some error in estimating concurrency. We used 2 different approaches as a check on internal validity of the simulation and found the approaches agreed well. Across the range of conditions we examined, concurrency status of partnerships "measured" with empirical levels of reporting error corresponded with the "true" temporal configuration of partnerships about $80 \%$ of the time.

As currently practiced in STD control programs, date elicitation is used for practical contact investigation purposes rather than for assessing concurrency. Because it is not designed for the purpose, much of the extra time and energy that might be devoted to memory aids, probes, and other techniques are not invested in the process. Similarly, the simulation used here to assess concurrency is not ideal. Several arbitrary decisions - length of lag, for example - may not be fully exploratory. The length of concurrency was not considered in this approach, nor was the interval between those partnerships that did not overlap. Because overlap is a surrogate for the actual events required for transmission (A has sex with $B$, then with $C$, then with $B$ again), a short duration of concurrency may not result in transmission, and a gap between partnerships may not prevent it. Finally, this simulation ignored the nonindependence of date reporting when 2 dyads have a person in common. Morris and O'Gorman also simulated the impact of error in reported dates on the measurement of concurrency. ${ }^{40}$ Although they implemented a different type of simulation model and did not use empirically based estimates of reporting error, their results were similar to ours in suggesting that the influence of reporting error on measured concurrency is mild to moderate.

In addition to the challenges of measuring concurrency with overlapping dates, different approaches for estimating concurrency may not be highly concordant. In 2 Seattle studies, the overlapping dates method correlated moderately with reported concurrency during the proximal sexual partnership (i.e., sex with other partners after date of first sex with the proximal partner) (kappas $=0.34$ $0.54)$ and strongly with reported concurrency involving the proximal partner and the second or third most proximal partner $(\kappa=$ $0.84)^{14}$ (MR Golden, unpublished data, personal communication to DDB, July 2002). Although the methods used in these studies differ from those typically used in contact tracing, the results from both studies suggest a significant discrepancy between measures of concurrency. Furthermore, measures of concurrency based on reports of the temporal ordering of encounters with partners are also likely to involve some error because the accuracy of reported order of autobiographic events, although good, is far from perfect. ${ }^{35}$

Clearly, a more rigorous qualitative and quantitative approach to understanding concurrency is required. ${ }^{7-9}$ Systematic research is also needed on the impact of forgetting partners ${ }^{41-43}$ on the measurement of concurrency, memory processes in generating reported dates, accuracy of reported exposure dates, individual-level correlates of reliability and validity, and interventions to enhance reporting of partnerships and their temporal properties.

\section{References}

1. Moody J. The importance of relationship timing in diffusion. Soc Forces 2002; 81:25-56 
2. Riolo CS, Koopman JS, Chick SE. Methods and measures for the description of epidemiologic contact networks. J Urban Health 2001; 78:446-457.

3. Kretzschmar M, Morris M. Measures of concurrency in networks and the spread of infectious disease. Math Biosci 1996; 133:165-195.

4. Morris M, Kretzschmar M. Concurrent partnerships and transmission dynamics in networks. Soc Netw 1995; 17:299-318.

5. Morris M, Kretzschmar M. Concurrent partnerships and the spread of HIV. AIDS 1997; 11:641-648.

6. Watts $\mathrm{CH}$, May RM. The influence of concurrent partnerships on the dynamics of HIV/AIDS. Math Biosci 1992; 108:89-104.

7. Gorbach PM, Stoner BP, Aral SO, et al. It takes a village: understanding concurrent sexual partnerships in Seattle, WA. Sex Transm Dis 2002; 29:453-462.

8. Gorbach PM, Drumright LN, Holmes KK. Discord, discordance, and concurrency: comparing individual and partnership-level analyses of new partnerships of young adults at risk of sexually transmitted infections. Sex Transm Dis 2005; 32:7-12.

9. Drumright LN, Gorbach PM, Holmes KK. Do people really know their sex partners? concurrency, knowledge of partner behavior, and sexually transmitted infections within partnerships. Sex Transm Dis 2004; 31:437-442.

10. Morris M. Concurrent partnerships and syphilis persistence: new thoughts on an old puzzle. Sex Transm Dis 2001; 29:504-507.

11. Potterat JJ, Zimmerman HP, Muth SQ, et al. Chlamydial transmission: concurrency, reproduction number and the epidemic trajectory. Am J Epidemiol 1999; 115:1331-1339.

12. Koumans EH, Farley TA, Gibson JJ, et al. Characteristics of persons with syphilis in areas of persisting syphilis in the United States: sustained transmission associated with concurrent partnerships. Sex Transm Dis 2001; 28:497-503.

13. Rothenberg RB, Potterat JJ, Gisselquist D. Concurrency and sexual transmission. AIDS 2002; 16:678-679.

14. Manhart LE, Aral SO, Holmes KK, et al. Sex partner concurrency: measurement, prevalence and correlates among urban 18-39-yearolds. Sex Transm Dis 2002; 29:133-143.

15. Centers for Disease Control and Prevention. Program operations: guidelines for STD prevention. Available at: http://www.cdc.gov/ std/program/partners.pdf.

16. Adimora AA, Schoenbach VJ, Bonas DM, et al. Concurrent sexual partnerships among women in the United States. Epidemiology $2002 ; 13: 320-327$.

17. Ghani AC, Ison CA, Ward H, et al. Sexual partner networks in the transmission of sexually transmitted diseases: an analysis of gonorrhea cases in Sheffield, UK. Sex Transm Dis 1996; 23:498-503.

18. Morris M. Sexual networks and HIV. AIDS 1997; 11:S209-S216.

19. Rothenberg RB, Morris M, Muth SQ, and the Network Studies Consortium. Large network concepts and small network characteristics. Available at: http://www.ima.umn.edu/complex/abstracts/11-17abs.html.

20. Potterat JJ, Rothenberg RB, Woodhouse DE, et al. Gonorrhea as a social disease. Sex Transm Dis 1985; 12:25-32.

21. Rothenberg RB, Potterat JJ. Temporal and social aspects of gonorrhea transmission: the force of infectivity. Sex Transm Dis 1988; 15:88-95.

22. Potterat JJ, Phillips-Plummer L, Muth SQ, et al. Risk network structure in the early epidemic phase of HIV transmission in Colorado Springs. Sex Transm Infect 2002; 78(suppl 1):i159-i163.

23. Zimmerman-Rogers H, Potterat JJ, Muth SQ, et al. Establishing efficient partner notification periods for patients with chlamydia. Sex Transm Dis 1999; 26:49-54.

24. Rothenberg RB, Sterk C, Toomey KE, et al. Using social network and ethnographic tools to evaluate syphilis transmission. Sex Transm Dis 1998; 25:154-160.

25. Centers for Disease Control and Prevention. Gang-related outbreak of penicillinase-producing Neisseria gonorrhoeae and other sexually transmitted diseases: Colorado Springs, 1989-1991. MMWR Morb Mortal Wkly Rep 1993; 42:25-28.

26. Thompson CP, Skowronski JJ, Larsen SF, et al. Autobiographical Memory: Remembering What and Remembering When. Mahwah, NJ: Lawrence Erlbaum Associates, Publishers, 1996.

27. Roberts JM Jr, Brewer DD. Measures and tests of heaping in discrete quantitative distributions. J Appl Stat 2001; 28:887-896.
28. Doreian P. Linear models with spatially distributed data: spatial disturbances or spatial effects? Soc Methods Res 1980; 9:29-60.

29. Dow MM. Model selection procedures for network autocorrelated disturbances models. Soc Methods Res 1986; 14:403-422.

30. Ord K. Estimation methods for models of spatial interaction. J Am Stat Assoc 1975; 70:120-126.

31. de Boer MA, Celentano DD, Tovanabutra S, et al. Reliability of self-reported sexual behavior in human immunodeficiency virus (HIV) concordant and discordant heterosexual couples in northern Thailand. Am J Epidemiol 1998; 147:1153-1161.

32. Lagarde E, Enel C, Pison G. Reliability of reports of sexual behavior: a study of married couples in rural West Africa. Am J Epidemiol 1995; $141: 1195-1200$.

33. van Duynhoven YTHP, Nagelkerke NJD, Van De Laar MJW. Reliability of self-reported sexual histories: test-retest and interpartner comparison in a sexually transmitted diseases clinic. Sex Transm Dis 1999; 26:33-42.

34. Goldstein MF, Friedman SR, Neaigus A, et al. Self-reports of HIV risk behavior by injecting drug users: are they reliable? Addiction 1995; 90:1097-1104.

35. Barclay CR, Wellman HM. Accuracies and inaccuracies in autobiographical memories. J Mem Lang 1986; 25:93-103.

36. Burt CDB. Retrieval characteristics of autobiographical memories event and date information. Appl Cogn Psychol 1992; 6:389-404.

37. Burt CDB, Kemp S, Conway M. What happens if you retest autobiographical memory 10 years on? Mem Cognit 2001; 29: $127-136$.

38. Rubin DC, Baddeley AD. Telescoping is not time compression: a model of the dating of autobiographical events. Mem Cognit 1989; 17:653-661

39. Thompson C. Memory for unique personal events: the roommate study. Mem Cognit 1982; 10:324-332.

40. Morris M, O'Gorman J. The impact of measurement error on survey estimates of concurrent partnerships. Math Pop Stud 2000; 8:231249.

41. Brewer DD, Potterat JJ, Muth SQ, et al. Randomized trial of supplementary interviewing techniques to enhance recall of sexual partners in contact interviews. Sex Transm Dis 2005; 32:189-193.

42. Brewer DD, Garrett SB, Kulasingam S. Forgetting as a cause of incomplete reporting of sexual and drug injection partners. Sex Transm Dis 1999; 26:166-176.

43. Brewer DD, Garrett SB. Evaluation of interviewing techniques to enhance recall of sexual and drug injection partners. Sex Transm Dis $2001 ; 28: 666-677$

\section{Appendix}

\section{Estimating the Effect of Unreliability in Reported Dates on} Estimation of Concurrency

The general layout for assessing concurrency depicts 2 dyads (sexual partnerships of durations $\mathrm{XZ}$ and QY), which we assume share 1 person in common:

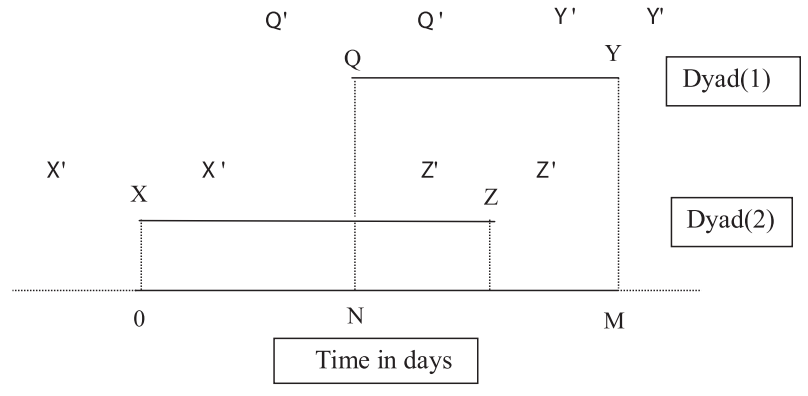

In this configuration, $\mathrm{XQ}$ is the lag between partnerships, $\mathrm{QZ}$ is the amount of concurrency (overlap), and we place $\mathrm{X}$ at time 0 for 
convenience. Though shown with $\mathrm{Q}>\mathrm{X}$ and $\mathrm{Y}>\mathrm{Z}$, the duration QY may stand in several different relationships to the duration $\mathrm{XZ}$ :

(1) If one dyad precedes another $(\{\mathrm{Q} \leq \mathrm{Y} \leq \mathrm{X} \leq \mathrm{Z}\}$ or $\{\mathrm{X} \leq$ $\mathrm{Z} \leq \mathrm{Q} \leq \mathrm{Y}\})$, then concurrency is given by $\mathrm{Y}-\mathrm{X}$ or $\mathrm{Z}-$ $\mathrm{Q}$, respectively, and is absent (negative).

(2) If one dyad partially overlaps another ( $\{\mathrm{Q} \leq \mathrm{X} \leq \mathrm{Y} \leq \mathrm{Z}\}$ or $\{\mathrm{X} \leq \mathrm{Q} \leq \mathrm{Z} \leq \mathrm{Y}\}$ ), then concurrency is $\mathrm{Y}-\mathrm{X}$ or $\mathrm{Z}-$ $\mathrm{Q}$, respectively, and is present (positive).

(3) If one dyad falls within the time frame of another ( $\{X \leq$ $\mathrm{Q} \leq \mathrm{Y} \leq \mathrm{Z}\}$ or $\{\mathrm{Q} \leq \mathrm{X} \leq \mathrm{Z} \leq \mathrm{Y}\})$, then concurrency is $\mathrm{Y}-\mathrm{Q}$ or $\mathrm{Z}-\mathrm{X}$, respectively, and is present (positive).

The potentially concurrent arrangements of $\{\mathrm{Q}=\mathrm{Y}=\mathrm{X}=\mathrm{Z}\}$, $\{\mathrm{Q}<\mathrm{Y}=\mathrm{X}<\mathrm{Z}\}$, and $\{\mathrm{X}<\mathrm{Z}=\mathrm{Q}<\mathrm{Y}\}$ did not occur in the simulations either as "true" or "observed" configurations (see below). This framework for assessing concurrency, like existing measures of concurrency, focuses on first and last exposure dates only and not the temporal patterning of encounters between such dates within a partnership.

Because the empirical distributions of differences in reported first and last exposure dates were distinctly nonnormal and not well described by other parametric distributions (e.g., log normal), we sampled from the empirical distributions. We used 2 approaches, each based on the notion that the disagreement between partners represents the actual level of reporting error, to generate dates $\left(\mathrm{X}^{\prime}, \mathrm{Z}^{\prime}, \mathrm{Q}^{\prime}\right.$, and $\left.\mathrm{Y}^{\prime}\right)$ that incorporate this error. The diagram shows a pair of possible positions for each of $\mathrm{X}^{\prime}, \mathrm{Z}^{\prime}, \mathrm{Q}^{\prime}$, and $\mathrm{Y}^{\prime}$ (error in reporting can either be forward or backward in time). The absolute and relative locations of such reported dates would vary in any particular simulation trial. $\mathrm{X}^{\prime}, \mathrm{Z}^{\prime}, \mathrm{Q}^{\prime}$, or $\mathrm{Y}^{\prime}$ can be left of 0 in the diagram merely due to the arbitrary placement of 0 .

In the first, a pair-based approach, we sampled 2 dyads randomly from all available dyads $(\mathrm{n}=723)$. For each dyad, we calculated the duration as the mean of the 2 members' reported durations and arbitrarily assigned $\mathrm{X}$ to zero. We then assigned a lag between them (XQ) by randomly sampling from a uniform distribution between $-\mathrm{N}$ and $+\mathrm{N}(+\mathrm{N}$ is shown in the diagram). Next, we added or subtracted, at random, the absolute difference in reported first exposure dates for that dyad to $X(Q)$, producing $X^{\prime}$ $\left(\mathrm{Q}^{\prime}\right)$, and added or subtracted, at random, the absolute difference in reported last exposure dates for that dyad to $\mathrm{Z}(\mathrm{Y})$, producing $\mathrm{Z}^{\prime}$ $\left(\mathrm{Y}^{\prime}\right)$. When these random alterations yielded "impossible" dates $\left(\mathrm{Z}^{\prime}<\mathrm{X}^{\prime}\right.$ or $\left.\mathrm{Y}^{\prime}<\mathrm{Q}^{\prime}\right)$, we set the duration $\left(\mathrm{X}^{\prime} \mathrm{Z}^{\prime}\right.$ or $\left.\mathrm{Q}^{\prime} \mathrm{Y}^{\prime}\right)$ to zero and randomly picked one of the dates as the partnership date. Despite the logical necessity of such corrections, the "observed" durations $\left(\mathrm{X}^{\prime} \mathrm{Z}^{\prime}\right.$ and $\left.\mathrm{Q}^{\prime} \mathrm{Y}^{\prime}\right)$ were $4 \%$ to $7 \%$ longer on average than the "true" durations (XZ and QY) for the different variations of the simulation presented in Table 3. At the end of a simulation trial, the presence or absence of concurrency in the initial (true) configuration was compared to concurrency in the altered (observed) configuration.

In the second, a distribution-based approach, we chose a "true" configuration by sampling a lag (XQ) as above and then sampled randomly from the distributions for duration in our data to assign lengths to $\mathrm{XZ}$ and QY (which also assign positions to all four temporal "nodes," with $\mathrm{X}$ arbitrarily assigned to zero). To obtain $\mathrm{X}^{\prime}$, we randomly selected one difference in reported dates of first exposure from the empirical distribution and added or subtracted (at random) this difference to $\mathrm{X}$. To obtain $\mathrm{Z}^{\prime}$, we randomly and independently selected one difference in reported dates of last exposure from the empirical distribution and added or subtracted (at random) this difference to Z. Parallel mechanisms yielded $\mathrm{Q}^{\prime}$ and $\mathrm{Y}^{\prime}$. Trials involving "observed" dyads with impossible dates were deleted and replaced with new trials involving valid dates. As above, we compared "true" concurrency to "observed" concurrency.

We performed multiple simulations with both approaches, each with 10,000 trials. These examined a range of possible subgroups, using lags of up to \pm 180 days and different levels of precision in reporting. For both approaches, we calculated sensitivity (proportion of truly concurrent configurations observed to be concurrent), specificity (proportion of truly not concurrent configurations observed to be not concurrent), positive predictive value (proportion of observed concurrent configurations that were truly concurrent), and concordance (the proportion of trials in which the observed concurrency matched the true concurrency status) with the true condition as the standard. 